\title{
Effect of magnetic frustration on single-hole spectral function in the $t-t^{\prime}-t^{\prime \prime}-J$ model
}

\author{
Y. Shibata, T. Tohyama and S. Maekawa \\ Institute for Materials Research, Tohoku University, Sendai 980-8577, Japan
}

(January 8, 2018)

\begin{abstract}
We examine the effect of the magnetic frustration $J^{\prime}$ on the single-hole spectral function in the $t-t^{\prime}-t^{\prime \prime}-J$ model. At zero temperature, the exact diagonalization (ED) and the self-consistent Born approximation (SCBA) methods are used. We find that the frustration suppresses the quasiparticle (QP) weight at small momentum $\mathbf{k}$, whereas the QP peak at $\mathbf{k}=(\pi / 2, \pi / 2)$ remains sharp. We also show the temperature dependence of the single-hole spectral function by using the ED method. It is found that the lineshapes at $(\pi / 2,0)$ and $(\pi / 2, \pi / 2)$ show different temperature dependence. These findings are consistent with the angle-resolved photoemission data on $\mathrm{Sr}_{2} \mathrm{CuO}_{2} \mathrm{Cl}_{2}$, and indicate the importance of the magnetic frustration on the electronic states of the insulating cuprates.

PACS numbers: 75.50.Ee, 78.20.Bh, 78.66.Nk, 79.60.-i
\end{abstract}

\section{INTRODUCTION}

Since the discovery of high temperature superconductivity, the $t-J$ model has been extensively studied as a theoretical model for the superconductors. However, angle-resolved photoemission spectroscopy (ARPES) measurements on $\mathrm{Sr}_{2} \mathrm{CuO}_{2} \mathrm{Cl}_{2}$ by Wells et al. $\mathrm{E}$ have clearly shown that the $t-J$ model does not explain the experimental data near $\mathbf{k}=(\pi, 0)$ : the $t-J$ model predicts that the energies of the quasiparticle $(\mathrm{QP})$ at $(\pi, 0)$ and $(\pi / 2, \pi / 2)$ are very similar, while the experimental energy at $(\pi, 0)$ is ical works 11 have shown that this discrepancy can be resolved by introducing hopping matrix elements to second and third nearest neighbors $\left(t^{\prime}\right.$ and $\left.t^{\prime \prime}\right)$ into the $t-J$ model. The Hamiltonian, termed the $t-t^{\prime}-t^{\prime \prime}-J$ model, is given by

$$
\begin{aligned}
H_{t t^{\prime} t^{\prime \prime} J}= & H_{t t^{\prime} t^{\prime \prime}}+H_{J}, \\
H_{t t^{\prime} t^{\prime \prime}}= & -t \sum_{\langle i, j\rangle_{1 \mathrm{st}} \sigma} \tilde{c}_{i \sigma}^{\dagger} \tilde{c}_{j \sigma}-t^{\prime} \sum_{\langle i, j\rangle_{2 \mathrm{nd}} \sigma} \tilde{c}_{i \sigma}^{\dagger} \tilde{c}_{j \sigma} \\
& -t^{\prime \prime} \sum_{\langle i, j\rangle_{3 \mathrm{rd}} \sigma} \tilde{c}_{i \sigma}^{\dagger} \tilde{c}_{j \sigma}+\text { H.c. } \\
H_{J}= & J \sum_{\langle i, j\rangle_{1 \mathrm{st}}} \mathbf{S}_{i} \cdot \mathbf{S}_{j}
\end{aligned}
$$

where $\tilde{c}_{i \sigma}=c_{i \sigma}\left(1-n_{i-\sigma}\right)$ is the annihilation operator of an electron with spin $\sigma$ at site $i$ with the constraint of no double occupancy, $\mathbf{S}_{i}$ is the spin operator and the summations $\langle i, j\rangle_{1 \mathrm{st}},\langle i, j\rangle_{2 \mathrm{nd}}$ and $\langle i, j\rangle_{3 \mathrm{rd}}$ run over first, second and third nearest-neighbor pairs, respectively.

Very recently, it has been argued 0 that not only the QP dispersion but also the lineshape of the ARPES data contains valuable information about the electronic states. It was shown that the $t-t^{\prime}-t^{\prime \prime}-J$ model describes the observed broad peak at $(\pi, 0)$. A weakening of the antiferromagnetic (AF) spin correlation induced by the charge-carrier motion due to the longe-range hoppings is found to be responsible for the broad lineshape.
The fact that the lineshape of ARPES spectra is sensitive to the AF correlation in the spin background suggests that, if magnetic frustration in the spin system is strong, the lineshape of the spectra should be also affected significantly. A dominant interaction which causes the magnetic frustration is considered to be the nextnearest neighbor exchange interaction $J^{\prime}$. The Hamiltonian is given by

$$
H_{J^{\prime}}=J^{\prime} \sum_{\langle i, j\rangle_{2 \mathrm{nd}}} \mathbf{S}_{i} \cdot \mathbf{S}_{j}
$$

The two-dimensional (2D) spin- $\frac{1}{2}$ Heisenberg Hamiltonian including both $J$ and $J^{\prime}$, i.e. $H_{J}+\mathrm{H}_{J^{\prime}}$, has been extensively studied, motivated by the suggestion ${ }^{12}$ that the physics of the high- $T_{c}$ phenomena is closely related to the presence of non-Néel states in insulating 2D cuprates. The phase diagram of the $J J^{\prime}$ model obtained as a function of $J^{\prime} / J$ is as follows: 13,14 the Néel state is the ground state for $0 \leq J^{\prime} / J \lesssim 0.6$, while a collinear state becomes stable in the ground state for $0.6 \lesssim J^{\prime} / J<1$. Since the realistic value of $J^{\prime} / J$ in the $2 \mathrm{D}$ insulating cuprates is expected to be $0.1 \sim 0.2,15-18$ the ground state remains to be the Néel state. Although the Néel state is stable, the excited states obtained after kicking photoelectron out of the system might be sensitive to the magnetic frustration. As far as we know there has been no study to examine the effect of $J^{\prime}$ on the single-hole spectral function.

In this paper, we examine the effect of the magnetic frustration $J^{\prime}$ on the single-hole spectral function at zero and finite temperatures in the $t-t^{\prime}-t^{\prime \prime}-J$ model. At zero temperature, the exact diagonalization (ED) and self-consistent Born approximation (SCBA) methods are used. The frustration is found to suppress the QP weight at small momentum. The suppression of the QP peak gives rise to a broad spectrum at the momentum, whereas the QP peak at $(\pi / 2, \pi / 2)$ remains sharp. This is consistent with the ARPES data on $\mathrm{Sr}_{2} \mathrm{CuO}_{2} \mathrm{Cl}_{2}$ that the $(\pi / 2,0)$ spectrum is broader than the $(\pi / 2, \pi / 2)$ one. We also calculate the single-hole spectral function at finite temperatures in the $t-t^{\prime}-t^{\prime \prime}-J$ model with $J^{\prime}$ by using the 
ED method. It is shown that the lineshapes between the $(\pi / 2,0)$ and $(\pi / 2, \pi / 2)$ spectra behave differently with increasing temperature. This difference is also consistent with experimental data by Kim and Shen.19 These findings indicate the importance of the magnetic frustration on the electronic states of the 2D insulating cuprates.

\section{METHOD OF CALCULATIONS}

The single-hole spectral function at temperature $T$ is given by

$$
\begin{aligned}
A(\mathbf{k}, \omega)= & \frac{1}{\mathcal{Z}} \sum_{m, n}\left|\left\langle\Psi_{n}^{N-1}\left|c_{\mathbf{k} \sigma}\right| \Psi_{m}^{N}\right\rangle\right|^{2} \\
& \times \delta\left(\omega-E_{m}^{N}+E_{n}^{N-1}\right) e^{-\beta E_{m}^{N}}
\end{aligned}
$$

where $\Psi_{m}^{N}$ is the wave function of the $m$-th eigenstates with energy $E_{m}^{N}$ in the $N$-electron system. $\mathcal{Z}=$ $\sum_{m} e^{-\beta E_{m}^{N}}$ is the partition function with $\beta=1 / k_{\mathrm{B}} T$. The Boltzmann factor $k_{\mathrm{B}}$ is taken to be 1 , hereafter. The initial states $\Psi_{m}^{N}$ are the eigenstates of the $J-J^{\prime}$ Heisenberg model. At $T=0$, the initial state is restricted to the ground state of the $J-J^{\prime}$ model. In the evaluation of Eq. (5) at finite temperatures, we use a $4 \times 4$ lattice with periodic boundary condition. After diagonalizing the $J$ $J$ Hamiltonian by the Householder method, we make use of the Lanczos procedure for each initial eigenstate in the Hamiltonian to obtain $A(\mathbf{k}, \omega)$.

At $T=0$, we also use the self-consistent Bornapproximation (SCBA) method to calculate $A(\mathbf{k}, \omega) 2022$ In the SCBA method, the hole Green's function $G(\mathbf{k}, \omega)$ and the self-energy $\Sigma(\mathbf{k}, \omega)$ satisfy a set of self-consistent equation for a $2 \mathrm{D}$ square lattice with $N$ sites:

$$
\begin{aligned}
G(\mathbf{k}, \omega) & =\frac{1}{\omega-\Sigma(\mathbf{k}, \omega)-\varepsilon_{\mathbf{k}}+i \eta} \\
\Sigma(\mathbf{k}, \omega) & =\sum_{\mathbf{q}} F(\mathbf{k}, \mathbf{q}) G\left(\mathbf{k}-\mathbf{q}, \omega-\omega_{\mathbf{q}}\right) \\
F(\mathbf{k}, \mathbf{q}) & =\frac{32 t^{2}}{N}\left|\gamma_{\mathbf{k}-\mathbf{q}} u_{\mathbf{q}}+\gamma_{\mathbf{k}} v_{\mathbf{q}}\right|^{2}
\end{aligned}
$$

where

$$
\begin{aligned}
& \varepsilon_{\mathbf{k}}=4 t^{\prime} \cos k_{x} \cos k_{y}+2 t^{\prime \prime}\left(\cos 2 k_{x}+\cos 2 k_{y}\right), \\
& \omega_{\mathbf{q}}=2 J \sqrt{\jmath_{\mathbf{q}}{ }^{2}-\gamma_{\mathbf{q}}^{2}}, \\
& \jmath_{\mathbf{q}}=1-\frac{J^{\prime}}{J}\left(1-\cos q_{x} \cos q_{y}\right), \\
& \gamma_{\mathbf{k}}=\frac{1}{2}\left(\cos k_{x}+\cos k_{y}\right) \\
& u_{\mathbf{q}}=\frac{1}{\sqrt{2}}\left(\sqrt{\frac{\jmath_{\mathbf{q}}{ }^{2}}{\jmath_{\mathbf{q}}{ }^{2}-\gamma_{\mathbf{q}}^{2}}}+1\right)^{\frac{1}{2}}, \\
& v_{\mathbf{q}}=-\operatorname{sign}\left(\gamma_{\mathbf{q}}\right) \frac{1}{\sqrt{2}}\left(\sqrt{\frac{\jmath_{\mathbf{q}}^{2}}{\jmath_{\mathbf{q}}{ }^{2}-\gamma_{\mathbf{q}}^{2}}}-1\right)^{\frac{1}{2}},
\end{aligned}
$$

and $1 \gg \eta>0$. The AF long-range order is assumed in the derivation of these equations. In the next section, we will compare the SCBA results with ED ones and see the validity of the assumption. $A(\mathbf{k}, \omega)$ is assumed to be equal to the imaginary part of the hole Green's function Eq. (7):

$$
A(\mathbf{k}, \omega)=-\frac{1}{\pi} \operatorname{Im} G(k, \omega)
$$
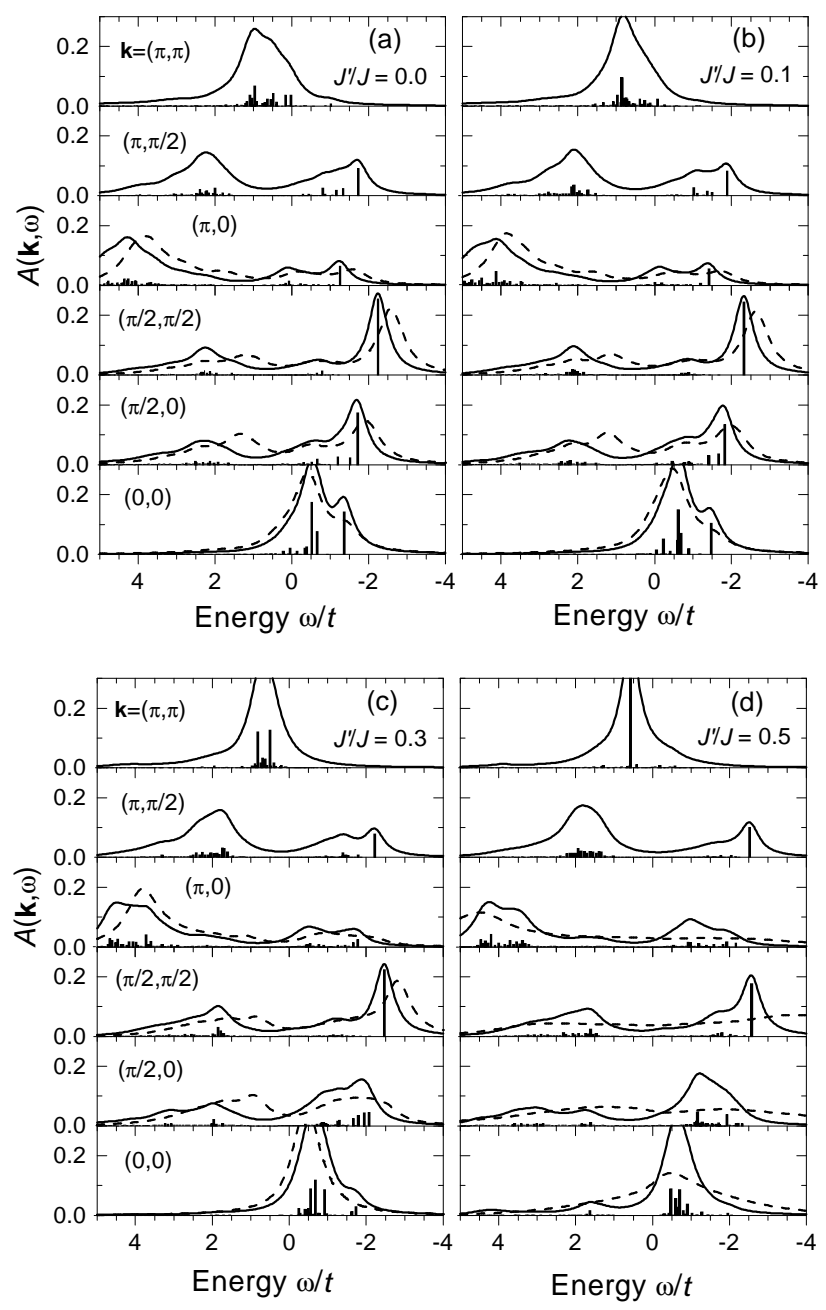

FIG. 1. Single-hole spectral function $A(\mathbf{k}, \omega)$ of the $t-t^{\prime}-t^{\prime \prime}-J$ model with various values of $J^{\prime}$ at $T=0 . t^{\prime}=-0.34 t$, $t^{\prime \prime}=0.23 t$ and $J=0.4 t . J^{\prime} / J$ is 0 (a), 0.1 (b), 0.3 (c), and 0.5 (d). Calculations are performed on the $4 \times 4$ cluster by the ED method (solid line) and the SCBA method (dashed line). The SCBA data at $(\pi, \pi)$ and $(\pi, \pi / 2)$ are excluded from the panels, since they are equivalent to those at $(0,0)$ and $(\pi / 2,0)$, respectively. The solid lines are obtained by introducing a Lorentzian broadening of $0.4 t$ into $\delta$-functions (vertical bars) in the ED results. 


\section{RESULTS AND DISCUSSIONS}

As mentioned in Sec. I, the nature of the ground state in the $J-J^{\prime}$ Heisenberg model changes from the Néel to collinear states at $J^{\prime} / J \sim 0.6$. 13, 14 By using the ED method, we found that the single-hole spectral function also shows different behavior between the two states.23 However, since we are interested in hole dynamics in the AF spin system with realistic values of $J^{\prime} / J$, we focus on the region $J^{\prime} / J<0.6$.

\section{A. Zero Temperature}

Figure 1 shows $A(\mathbf{k}, \omega)$ of the $t-t^{\prime}-t^{\prime \prime}-J$ model with various values of $J^{\prime}$ at $T=0$. Parameter values are set to be $J=0.4 t, t^{\prime}=-0.34 t$ and $t^{\prime \prime}=0.23 t$ as used in Ref. 2 . Solid lines and vertical bars are the ED results on a $4 \times 4$ cluster. In Fig. 1(a) $\left(J^{\prime} / J=0\right)$, the height of the QP peak $(\omega / t \sim-1.2)$ at $(\pi, 0)$ is small as compared with those at $(\pi / 2 \pi / 2)$ and $(\pi / 2,0) \quad(\omega / t \sim-2.2$ and -1.7 , respectively). Since $t^{\prime}$ and $t^{\prime \prime}$ terms cause the shift of the $\mathrm{QP}$ peak at $(\pi, 0)$ to higher binding energy, the position of the QP peak enters into the region where the incoherent states exist. As a result, the QP weight is strongly suppressed.

With increasing $J^{\prime}$ the ED results show the decrease of the QP weight for all the momenta. In particular, the remarkable decrease is found at $(0,0)$ and $(\pi / 2,0)$. The spectrum near the QP energy at $(\pi / 2,0)$ becomes broad as clearly seen in Figs. 1(c) and (d). Such a broad spefi trum has been observed at $(\pi / 2,0)$ in ARPES datal 10 Since the QP peak without $J^{\prime}$ is as sharp as that at $(\pi / 2, \pi / 2)$ (see Fig. 1(a)), the observed broad spectrum is considered to be a manifestation of the effect of frustration due to $J^{\prime}$.

In order to clarify the effect of $J^{\prime}$ on the spectral function, we also use the SCBA method for the calculation of $A(\mathbf{k}, \omega)$. In Fig. 1, the SCBA results are compared with the ED ones to see the validity of the approximation. The SCBA data (dashed lines) are plotted for momenta inside the magnetic first Brillouin zone. The spectra obtained by the SCBA method are in good agreement with those by the ED one, except for $J^{\prime} / J=0.5$ where the Néel state is expected to be less stable in the ground state. Encouraged by the good agreement between the ED and SCBA results at $J^{\prime} / J \leq 0.3$, we extend the size of cluster for the SCBA method from $4 \times 4$ to $16 \times 16$. Figure 2 shows the SCBA results at various momenta for the $16 \times 16$ cluster. Shown in the figure are $A(\mathbf{k}, \omega)$, the imaginary part of the self-energy $\operatorname{Im} \Sigma(\mathbf{k}, \omega)$ and the $\mathrm{QP}$ weight $Z\left(\mathbf{k}, \varepsilon_{\mathrm{QP}}\right)$ defined as

$$
Z\left(\mathbf{k}, \varepsilon_{\mathrm{QP}}\right)=\left(1-\left.\frac{\partial \operatorname{Re} \Sigma(\mathbf{k}, \omega)}{\partial \omega}\right|_{\omega=\varepsilon_{\mathrm{QP}}}\right)^{-1}
$$

where $\varepsilon_{\mathrm{QP}}$ is the QP energy at $\mathbf{k}$.
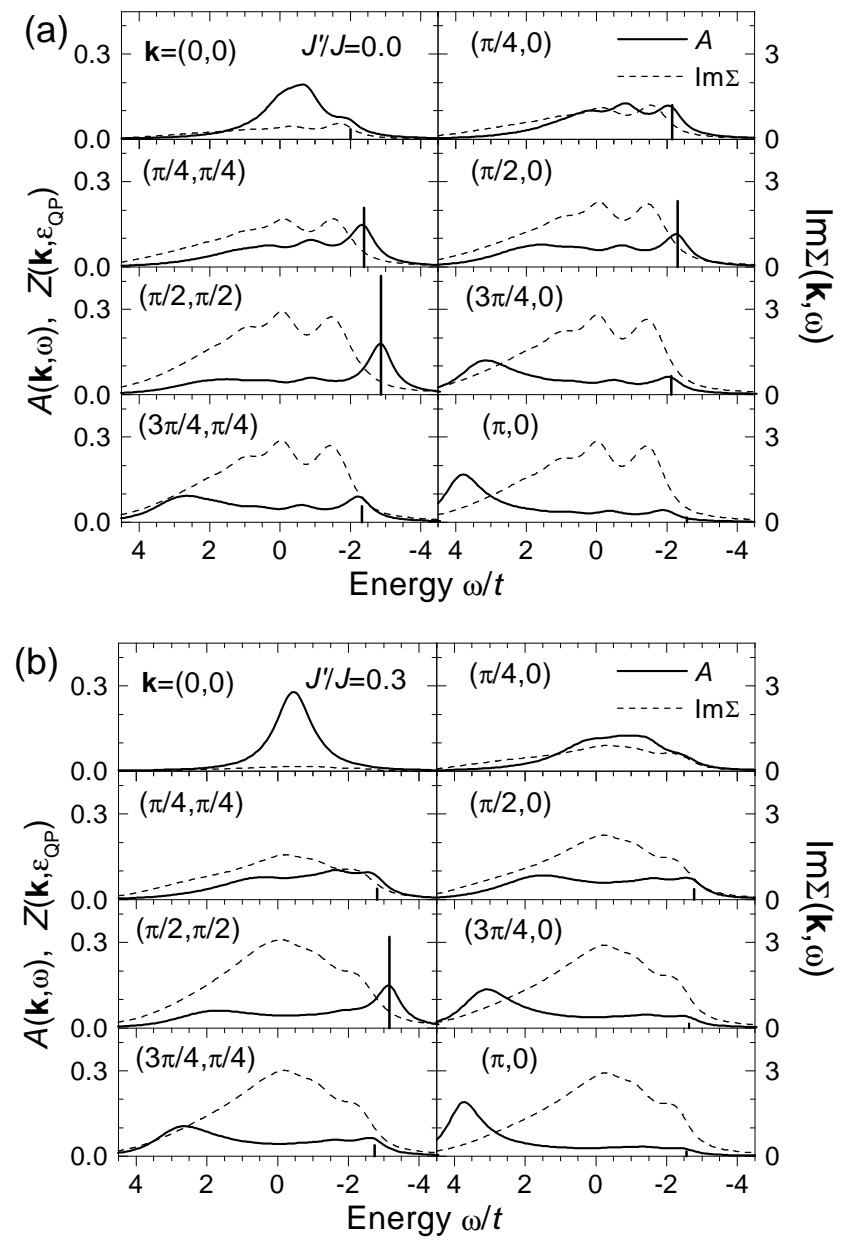

FIG. 2. Single-hole spectral function $A(\mathbf{k}, \omega)$ (solid line), the imaginary part of the self-energy $\operatorname{Im} \Sigma(\mathbf{k}, \omega)$ (dashed line) and the QP weight $Z\left(\mathbf{k}, \varepsilon_{\mathrm{QP}}\right)$ (vertical line) of the $t-t^{\prime}-t^{\prime \prime}-J$ model with $J^{\prime}$ at $T=0$ obtained by the SCBA method on a $16 \times 16$ cluster. $t^{\prime}=-0.34 t, t^{\prime \prime}=0.23 t$ and $J=0.4 t . \quad J^{\prime} / J$ is 0 (a) and 0.3 (b). $\eta / t=0.4$ for $A(\mathbf{k}, \omega)$ and $\operatorname{Im} \Sigma(\mathbf{k}, \omega)$, and $\eta / t=0.005$ for $Z\left(\mathbf{k}, \varepsilon_{\mathrm{QP}}\right)$. In $(\mathrm{b}), Z$ at $(0,0)$ and $(\pi / 4,0)$ is zero.

With increasing $J^{\prime} / J$ from 0 (Fig. 2(a)) to 0.3 (Fig. 2(b)), the spectral intensity around the QP energy becomes broader when the momentum $\mathbf{k}$ is close to $(0,0)$. Since the life time of the QP expressed by $\operatorname{Im} \Sigma(\mathbf{k}, \omega)$ shows little change, the broadness comes from the decrease of the QP weight $Z$. For $\mathbf{k}=(0,0)$ and $(\pi / 4,0), Z$ is zero at $J^{\prime} / J=0.3$. On the other hand, for large momenta such as $(3 \pi / 4, \pi / 4)$ and $(\pi, 0)$, the change of $Z$ is very small. In the intermediate values of $\mathbf{k}$, the ratio of $Z$ for $J^{\prime} / J=0.3$ to that for $J^{\prime} / J=0$ decreases as $\mathbf{k}$ approaches $(0,0)$ point. The maximum value of the ratio appears at $(\pi / 2, \pi / 2)$. Similar $\mathbf{k}$ dependent behaviors of $Z$ are obtained when the value of $J$ in the $t-J$ model is reduced.22 The similarity between the effects due to $J$ in the $t$ - $J$ model and $J^{\prime}$ in our model is also seen in the dependence of the QP band width $W$, i.e. with increasing 
$J^{\prime}, W$ decreases monotonically, which resembles a wellknown behavior of $W \propto J$ in the $t-J$ model 24 Therefore, we can say that the changes of $A(\mathbf{k}, \omega)$ due to the increase of $J^{\prime}$ are roughly similar to those due to the decrease of $J$ in the $t-J$ model. This is consistent with an intuitive picture that $J^{\prime}$ frustrate the Néel state as if the net value of $J$ is reduced.
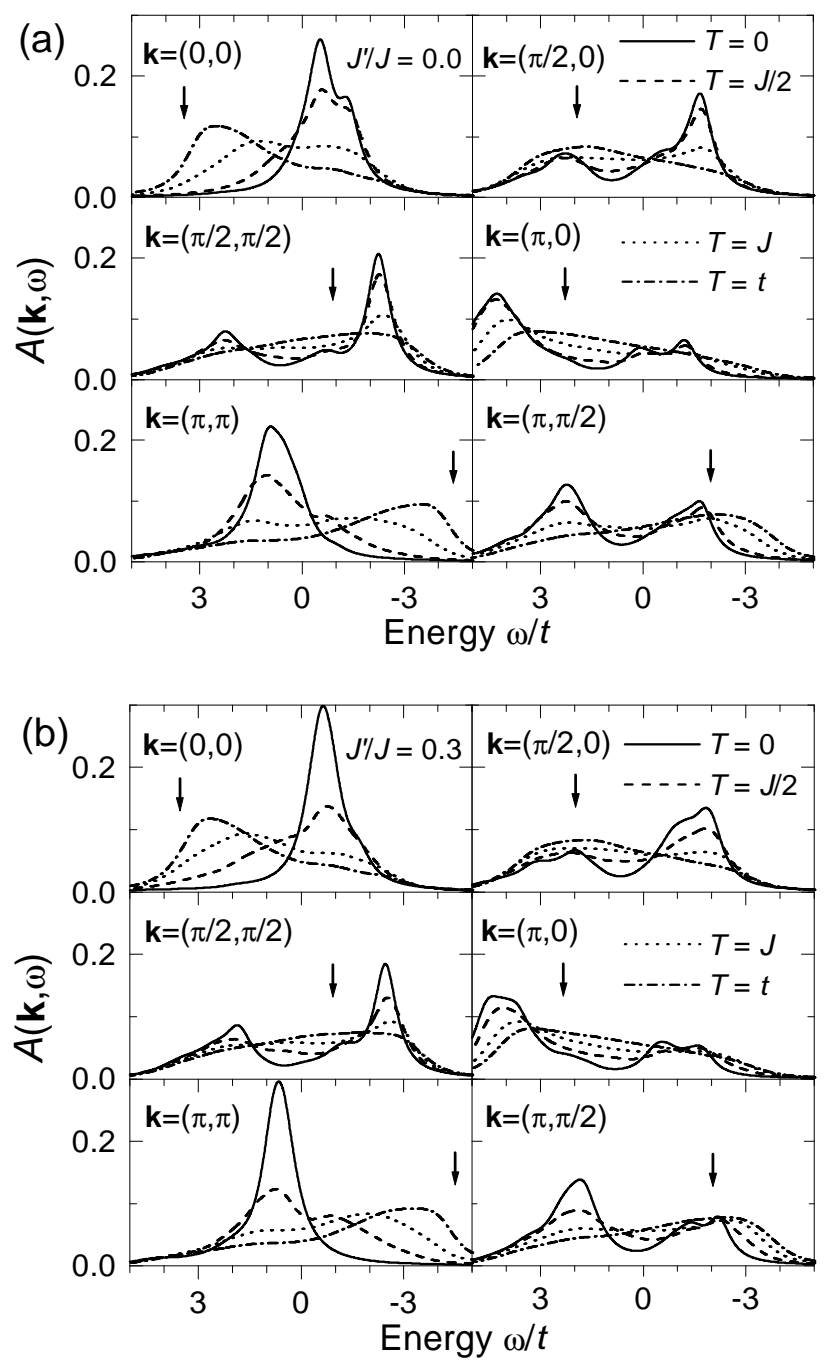

FIG. 3. Single-hole spectral function $A(\mathbf{k}, \omega)$ of the $t-t^{\prime}-t^{\prime \prime}-J$ model with $J^{\prime}$ at various temperatures. $t^{\prime}=-0.34 t$, $t^{\prime \prime}=0.23 t$ and $J=0.4 t . \quad J^{\prime} / J$ is 0 (a) and $0.3(\mathrm{~b})$. The values of temperature are $T=0$ (solid line), $J / 2$ (dashed line), $J$ (dotted line) and $t$ (dot-dashed line). Calculations are performed on the $4 \times 4$ cluster by the ED method. A Lorentzian broadening of $0.4 t$ is introduced into the $\delta$-functions.

\section{B. Finite Temperatures}

We next present the temperature dependence of $A(\mathbf{k}, \omega)$ on a $4 \times 4$ lattice. Since the energy separation between the ground and first excited states in the $J$ -
$J^{\prime}$ Heisenberg model on the lattice is roughly $J / 2$, reliable results are obtained only in the region of $T \gtrsim J / 2$. Although the temperature one can treat is rather high, the effect of the change of spin correlation length $\xi$ on the spectrum may be seen in the calculation because the correlation length $\xi$ for the temperatures above $T \sim J / 2$ is less than the maximum length of the $4 \times 4$ lattice $(\sim 2 \sqrt{2} a)$.25

Figure 3 shows $A(\mathbf{k}, \omega)$ of the $t-t^{\prime}-t^{\prime \prime}-J$ model with $J^{\prime} / J=0$ and 0.3 at various temperatures obtained by using the ED method. The QP spectral intensity at $(\pi / 2, \pi / 2)$ decreases with increasing temperature. This is not due to the decrease of the $\mathrm{QP}$ weight but due to the decrease of the life time.26 We find that, when the temperature changes from $T=0$ to $J / 2$, the intensity in Fig. 3(b) $\left(J^{\prime} / J=0.3\right)$ is largely suppressed as compared with that in Fig. 3(a). This simply comes from the fact that the frustration $J^{\prime}$ disturbs the AF spin background which is responsible for the formation of $\mathrm{QP}$ and makes the background sensitive to a slight change in temperature. Such a sensitive behavior of the QP peak may be one of the origins of the ghserved strong temperature dependence of ARPES data.19

In Fig. 3(b), we find that the dependence of the spectra near the QP energy on temperature at $(\pi / 2, \pi / 2)$ is remarkably different from that at $(\pi / 2,0)$. At $(\pi / 2, \pi / 2)$ the spectral weight around $\omega \sim-t$ slightly increases with increasing temperature in spite of the reduction of the QP intensity. On the contrary, the weight in the same energy region at $(\pi / 2,0)$ is uniformly suppressed with increasing temperature. Such a different behavior has been observed in ARPES experiment on $\mathrm{Sr}_{2} \mathrm{CuO}_{2} \mathrm{Cl}_{2}$. 19 . Since the behavior can not be clearly seen without $J^{\prime}$ (see Fig. 3(a)), the agreement between theory and experiment indicates the importance of $J^{\prime}$ on the electronic states of insulating cuprates.

With increasing temperature from $T=0$ to $J$, the QP spectral intensity in Figs. 3(a) and (b) rapidly decreases accompanied by a slight shift to lower-energy side.27 29 At $T=J$, the QP peaks almost vanish because the correlation length $\xi$ is less than one-lattice spacing 25 Similar suppression of the weight as the temperature increases from $T=0$ to $J$ is seen in the other peaks at $\omega \sim 0, t, 2 t$ and $4 t$ for $(0,0),(\pi, \pi),(\pi, \pi / 2)$ and $(\pi, 0)$, respectively. The weight at these peaks is thus sensitive to the nature of the spin background. At $T=t$, the spectrum has a broad maximum at a certain energy in each momentum. This behavior is independent of the value of $J^{\prime}$. In such a high temperature region, the hole can propagate without being disturbed by the spin background. 30 This produces a dispersive structure whose energy is determined by a tight-binding band due to the free hole propagation. The energy band is given by

$$
\begin{aligned}
E_{\text {free }}(\mathbf{k})= & 2 t\left(\cos k_{x}+\cos k_{y}\right)+4 t^{\prime} \cos k_{x} \cos k_{y} \\
& +2 t^{\prime \prime}\left(\cos 2 k_{x}+\cos 2 k_{y}\right) .
\end{aligned}
$$

In Fig. 3, the energy obtained from the band is indicated 
by arrow at each $\mathbf{k}$ point. With further increasing temperature, the maximum of the spectrum is expected to shift to the position indicated by the arrow.

\section{CONCLUSIONS}

We have examined the effect of the magnetic frustration $J^{\prime}$ on the single-hole spectral function in the $t-t^{\prime}-t^{\prime \prime}$ $J$ model, by using the ED and SCBA methods. At zero temperature, the frustration suppresses the QP weight and makes the spectrum broad for small momentum, whereas the peak at $(\pi / 2, \pi / 2)$ remains sharp. Our results explain the experimental data that the $(\pi / 2,0)$ spectrum in $\mathrm{Sr}_{2} \mathrm{CuO}_{2} \mathrm{Cl}_{2}$ is broader than the $(\pi / 2, \pi / 2)$ one. We have also evaluated the temperature dependence of the single-hole spectral function in the $t-t^{\prime}-t^{\prime \prime}-J$ model with $J^{\prime}$ by using the ED method. The lineshape between $(\pi / 2,0)$ and $(\pi / 2, \pi / 2)$ spectra behaves differently with increasing temperature. This difference is also consistent with recent experimental data. These findings indicate the importance of the magnetic frustration on the electronic states of the insulating cuprates.

\section{ACKNOWLEDGMENTS}

We would like to thank C. Kim and Z.-X. Shen for sharing with their experimental data prior to publication and for stimulating discussions. We also thank P. Prelovšek for informing us Ref. 26. This work was supported by CREST and NEDO. The numerical calculations were performed in the Supercomputer Center, Institute for Solid State Physics, University of Tokyo, and the supercomputing facilities in Institute for Materials Research, Tohoku University.

${ }^{1}$ B. O. Wells, Z.-X. Shen, A. Matsuura, D. M. King, M. A. Kastner, M. Greven, and R. J. Birgeneau, Phys. Rev. Lett. 74, 964 (1995).

${ }^{2}$ C. Kim, P. J. White, Z.-X. Shen, T. Tohyama, Y. Shibata, S. Maekawa, B. O. Wells, Y. J. Kim, R. J. Birgeneau, and M. A. Kastner, Phys. Rev. Lett. 80,4245 (1998).

${ }^{3}$ A. Nazarenko, K. J. E. Vos, S. Haas, E. Dagotto, and R. Gooding, Phys. Rev. B 51, 8676 (1995).

${ }^{4}$ B. Kyung and R. A. Ferrell, Phys. Rev. B 54, 10125 (1996).

${ }^{5}$ T. Xiang and J. M. Wheatley, Phys. Rev. B 54, R12653 (1996).

${ }^{6}$ V. I. Belinicher, A. L. Chernyshev, and V. A. Shubin, Phys. Rev. B 5414914 (1996).

${ }^{7}$ T. K. Lee and C. T. Shih, Phys. Rev. B 55, 5983 (1997).

${ }^{8}$ R. Eder, Y Ohta, and G. A. Sawatzky, Phys. Rev. B 55, R3414 (1997).
${ }^{9}$ P. W. Leung, B. O. Wells, and R. J. Gooding, Phys. Rev. B 56, 6320 (1997).

${ }^{10}$ F. Lema and A. A. Aligia, Phys. Rev. B 55, 14092 (1997).

${ }^{11}$ D. Duffy, A. Nazarenko, S. Haas, A. Moreo, J. Riera, and E. Dagotto, Phys. Rev. B 56, 5597 (1997).

${ }^{12}$ P. W. Anderson, Science 235, 1196 (1987).

${ }^{13}$ F. Mila, D. Poilblanc, C. Bruder, Phys. Rev. B 43, 7891 (1991).

14 S. Miyazawa and S. Homma, Phys. Lett. A 193, 370 (1994).

15 T. Tohyama and S. Maekawa, J. Phys. Soc. Jpn. 59, 1760 (1990).

${ }^{16}$ H. Eskes, L. F. Feiner and G. A. Sawatzky, Physica C 160, 424 (1989)

17 J. F. Annett, R. M. Martin, A. K. McMahan and S. Satpathy, Phys. Rev. B 40, 2620 (1989).

${ }^{18}$ D. K. Morr, Phys. Rev. B 58, R587(1998).

${ }^{19}$ C. Kim and Z.-X. Shen, private communication.

${ }^{20}$ S. Schmitt-Rink, C. M. Varma, and A. E. Ruckenstein, Phys. Rev. Lett. 60, 2793 (1988).

${ }^{21}$ C. L. Kane, P. A. Lee and N. Read, Phys. Rev. B 39, 6880 (1989).

22 G. Martinez and P. Horsh, Phys. Rev. B 44, 317 (1991).

${ }^{23}$ Y. Shibata, T. Tohyama and S. Maekawa, unpublished.

${ }^{24}$ E. Dagotto, Rev. Mod. Phys. 66, 763 (1994); and references therein.

${ }^{25}$ H.-Q. Ding and M. S. Makivić, Phys. Rev. Lett., 64, 1449 (1990).

${ }^{26}$ J. Jaklič, thesis, University of Ljubljana (1996).

27 J. Igarashi and P. Fulde, Phys. Rev. B 48, 998 (1993).

${ }^{28}$ J. van den Brink and O. P. Sushkov, Phys. Rev. B 57, 3518 (1998).

29 J. Jaklič and P. Prelovšek, cond-mat/9803331 (to be publised in Adv. Phys.).

${ }^{30}$ W. F. Brinkman and T. M. Rice, Phys. Rev. B 2, 1324 (1970). 